models should include the important coupling between chemistry, radiation and dynamics. Model calculations of ozone depletion were shown to be sensitive to these feedback mechanisms.

The problem of just what effect an increase in ultraviolet radiation might have on the population was discussed by R. H. Mole of the Medical Research Council Radiobiology Unit, Harwell. He found faults with a wholly uncritical acceptance of the correlation between latitude and occurrence of skin cancer. Changing social conventions in dress and increasing mobility of the population are certainly likely to be very important factors.

In summary, the symposium in reviewing recent work highlighted many uncertainties. There is clearly a need for a better understanding of the chlorine budget, the continuing measurement programme being very important. Further improvements in numerical models are necessary, with greater emphasis on the meteorology of the problem. Finally, it would be of great help to the planners who must make some decision on the use of CFCs if a more reliable understanding of the possible medical effects of an increase in ultraviolet radiation dosages were gained.

\section{Microscopic optical potentials}

\author{
from P. E. Hodgson
}

AN international workshop on microscopic nuclear optical potentials was held at the University of Hamburg from 25 to 27 September.* Organised by Professor H. V. von Geramb, it attracted over 100 participants and provided a welcome opportunity to review the recent progress in calculating optical potentials from the nucleonnucleon interaction. Powerful methods of calculating the optical potential for finite nuclei have been developed by Jeukenne, Lejeune and Mahaux in Liège and by Rook and Brieva in Oxford (Nature 253, 163; 1975 and 269, $750 ; 1977)$. The resulting potentials fit the data quite well, without further parameter adjustment. This opens the way to a detailed understanding of the interaction of nucleons and groups of nucleons with nuclei, assists the phenomenological analyses of experimental data, and should stimulate further accurate experimental work. The latest developments in these calculations

*A summary of the workshop is available on request from $P$. E. Hodgson. The papers will be published by Springer Verlag as
Lecture Notes in Physics series. were reviewed by $\mathrm{C}$. Mahaux and by F. A. Brieva, and related work by $\mathrm{I}$. McCarthy (Adelaide) and M. Weigel (Munich).

At lower energies, the structure of the target nucleus affects the interaction, and the effects of the coupling to the single-particle and collective states may be included in the calculation of the imaginary part of the potential by explicit summation over intermediate states. Work of this type was described by V. A. Madsen (Oregon), N. Vinh Mau (Orsay) and Van Giai (Orsay), and applied to nucleon and alphaparticle scattering from ${ }^{40} \mathrm{Ca}$ and ${ }^{208} \mathrm{~Pb}$.

It is now clear that the Saxon-Woods form factor universally used in optical model calculations is not always a sufficiently accurate representation of the potential. Improvements can be made by using microscopic form factors, or by supplementing the potential by an extra term depending on the orbital angular momentum $L$, or by altering the shape of the potential in a systematic way to fit the experimental data. A microscopic basis for an improved potential was provided by Y. C. Tang (Minnesota) who used the resonating group formalism to show that antisymmetrising the wavefunctions in a folding model calculation gives rise to parity-dependent terms in the potential. These take account of exchange processes and become less important as the mass difference between the interacting particles increases.

In some interactions there is a significant difference between the optical model predictions and the experimental data, and there is an obvious physical reason. It is then possible either to incorporate the physical effect in the calculations, or to add an extra phenomenological term to the potential. An example of this is the strong coupling to low-lying collective states which in the case of sub-Coulomb scattering of heavy ions causes the cross section to depart from the usual Fresnel form. This was discussed by A. J. Baltz (Brookhaven), who showed that the data can be fitted by the addition of a long-range dynamic absorption potential that can be obtained in closed form. Further analysis of this process was presented by G. Baur (Jülich). Another example is provided by the strong coupling to particular reaction channels. This alters the elastic cross section in a way that can be fitted by an $L$-dependent potential, as shown by Mackintosh (Daresbury). He also showed the value of model-independent analyses of elastic scattering.

Extensive work on the unification of the bound state and scattering potentials was presented by M. M. Giannini (Genova), and other global phenomenological analyses by A. Tarrats (Saclay) and by H. Leeb (Vienna). A new method of measuring cross section ratios was described by S. M. Austin (Michigan) and extensive measurements of proton scattering by light nuclei by M. Pignanelli (Milano).

The analysis of the scattering of composite particles by nuclei has not yet been developed to the same stage of sophistication as nucleon scattering. Several folding models give semi-phenomenological potentials that often fit the data quite well, but much of the important physics, especially the effects of antisymmetrisation and exchange, is still hidden in the adjustable normalisation parameter. A pioneer study of deuteron interactions was presented by A. A. Ionnides (Surrey) but apart from this the development of microscopic potentials for composite particles is still in the future. Meanwhile, a wealth of accurate data on the elastic scattering of deuterons and alpha-particles was presented by several groups, and in most cases they are very well fitted by optical model analyses.

Calculations of the imaginary part of the optical potential for heavy ions were reviewed by D. M. Brink (Oxford) who also described a new approach using the Feynmann path integral method. This can be used to relate the imaginary potential to the friction coefficient in heavy-ion scattering. D. F. Jackson (Surrey) showed how the extreme sensitivity of the alpha-decay rate to the potential barrier can be used to define the optical potential more precisely. A repulsivecore alpha-particle potential that gives the energies and widths of unbound cluster states and also fits the elastic scattering was described by K. A. Gridnev (Leningrad). Molecular states in heavy ion potentials were reviewed by W. Scheid (Giessen).

A fully microscopic description of heavy-ion reactions is not yet practicable, but considerable progress has been made using the time-dependent Hartree-Fock method. P. G. Reinhard (Mainz) has used this method to determine the collective Hamiltonian and hence the quantum corrections to the optical potential.

The meeting showed the extent and vitality of studies of all aspects of optical model potentials. The techniques for calculating microscopic potentials have now been established for nucleons, and much work remains to be done to improve the calculation of small terms and to apply the method to a wide range of nuclei and energies. At the same time it will be extended to composite particles, to link up with the very extensive phenomenological analyses that have already been made.

P. E. Hodgson is in the Department of Nuclear Physics at the University of Oxford. 